\title{
A new technique for assessing fixation-induced morphological variation in octopus (Mollusca: Cephalopoda)
}

\author{
STEVE O'SHEA \\ National Institute of Water and Atmospheric \\ Research Ltd \\ P.O. Box 14-901 Kilbirnie \\ Wellington, New Zealand
}

\begin{abstract}
The morphology of a preserved octopod is significantly affected by the condition of the animal at the time of fixation, whether live or dead. When unknown, this condition can be assessed by examining the degree of contraction of the radula support muscle. Specimens fixed post-mortem have a relaxed radula support muscle. This muscle is contracted in specimens plunged into preservatives alive. The degree of contraction of this radula support muscle facilitates identification of both fixationinduced and fixation-independent character states in octopod systematics.
\end{abstract}

Keywords fixation; character states; radula support muscle; Mollusca; Octopoda; systematics

\section{INTRODUCTION}

The New Zealand octopod fauna currently is being revised by the author; the systematic research is based primarily upon extensive collections of preserved specimens. The two principal sources of cephalopod material for New Zealand museum collections are specimens collected by research expeditions and specimens collected as incidental bycatch from the commercial fishing industry. Specimens collected in the course of research activities generally have been fixed live, whereas commercial bycatch material is typically frozen and fixed postmortem when received at the museum. Furthermore,

Received 13 June 1995; accepted 25 November 1996 specimens collected intentionally are, in general, juvenile or small bodied, whereas those collected as bycatch tend to be either adult or large bodied. Consequently, the relationship between adult and juvenile specimens of the same species subjected to different fixation histories may be obscured. This is particularly a problem with relatively rare or new taxa.

The morphology of a preserved octopod is significantly affected by the state of the animal during the process of fixation, whether the animal was fixed live or post-mortem. Specimens fixed while alive appear squat bodied, short armed, and highly papillose (Fig. 1). Specimens fixed post-mortem have longer, straight arms, smoother skins and either slumped or slender-profiled bodies (Fig. 2). When numerous specimens are available for comparison, variation attributable to fixation history usually can be identified. The relationship between various fixation-induced morphologies, however, is complicated for rare species such as deep-sea taxa, many of which are naturally squat bodied, short armed, and smooth skinned. As systematic reviews of octopods usually involve examination of preserved specimens, for which the initial fixation history is seldom recorded, it has proven necessary to differentiate between fixation-induced and fixation-independent character states. The procedure outlined here permits identification of the condition of a specimen at the time of fixation, and may find wider application in other systematic reviews of octopods based on preserved specimens.

\section{METHODS}

The two specimens of Thaumeledone sp. illustrated (Fig. 1, 2) are from collections of the National Institute of Water and Atmospheric Research (collection acronym NZOI) with accession and locality data as follows: Fig. 1 - NZOI Stn S153, female, ML $37.2 \mathrm{~mm}, 45^{\circ} 21.1^{\prime} \mathrm{S}, 173^{\circ} 35.8^{\prime} \mathrm{E}, 1386 \mathrm{~m}, 27 \mathrm{Oct}$ 1979; and Fig. 2 - NZOI Stn Z8393, male, ML $45.5 \mathrm{~mm}, 44^{\circ} 13.43-14.00^{\prime} \mathrm{S}, 178^{\circ} 41.59-44.29^{\prime} \mathrm{E}$, 


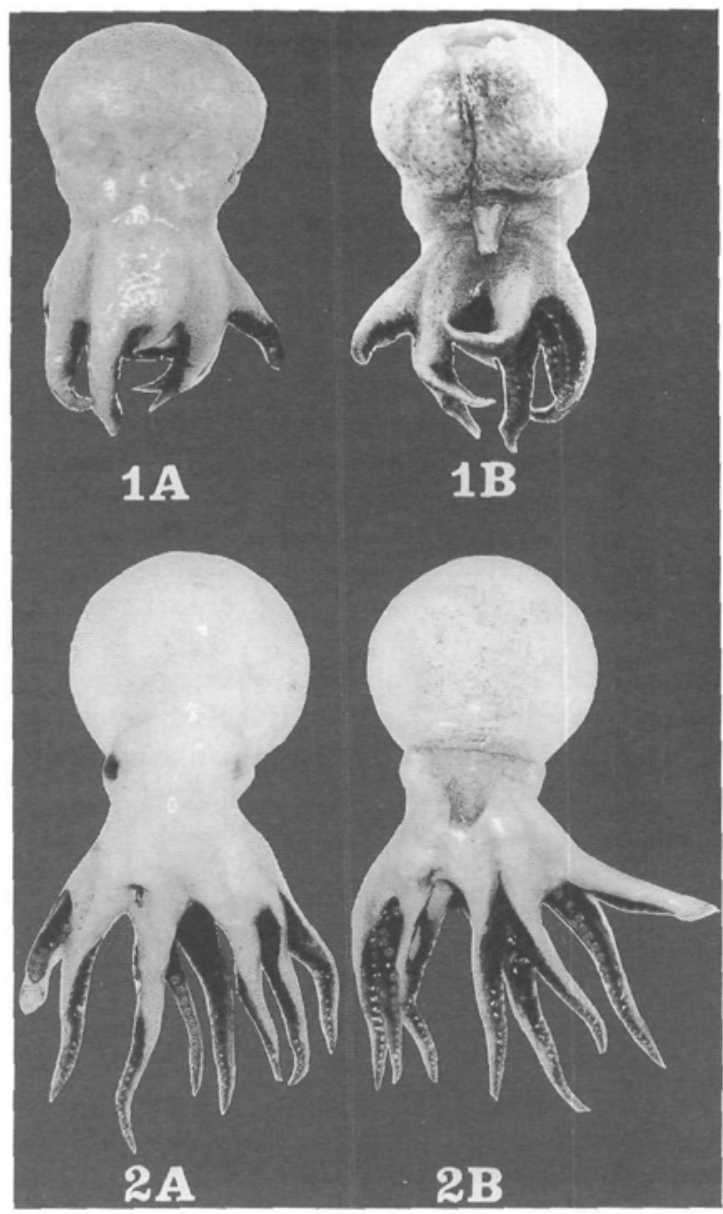

Fig. 1,2 Thaumeledone sp., whole animal views, dorsal (A) and ventral (B): 1, NZOI Stn S153, submature female fixed live; 2, NZOI Stn Z8393, mature male fixed postmortem (post-thaw).

1066-1073 m, 12 Oct 1995, f.r.v. Tangaroa Stn TAN9511/042. Both specimens have known treatment history: NZOI Stn S153 was fixed live, NZOI Stn Z8393 was fixed post-mortem (post-thaw).

The terminology of the buccal mass employed here is based on that used for Octopus (Nixon 1980). To remove the buccal mass from the head, dissect through the arm crown through web sector D (Roper \& Voss 1983), then through the connective tissue holding the buccal mass in place and the oesophagus. After the buccal mass is dissected from the head of the octopod, the buccal musculature that holds the beaks in situ can be cut and the beaks extracted. This exposes both the salivary papilla and the lateral buccal palps (Fig. 3). An off-midline sagittal section is then made through the buccal mass to expose the radula, radula sac, and radula support muscle (Fig. 4), the latter running from the base of the buccal complex to the base of the exposed portion of the radula.

\section{RESULTS}

Contraction of the radula support muscle depresses the position of the radula sac, and a section of exposed radula, relative to the lateral buccal palps and salivary papilla. If a specimen was fixed live, the radula ribbon will be withdrawn into the buccal mass (Fig. 3), and the radula support muscle will be appreciably contracted (Fig. 4). If a specimen is fixed post-mortem, the radula will be relatively exposed (Fig. 5), and the radula support muscle will be relaxed (Fig. 6).

Presumably the radula support muscle contracts in concert with other body musculature if a specimen is stressed during fixation (e.g. when an animal is fixed live). In material fixed post-mortem, all muscles are entirely relaxed.

\section{DISCUSSION}

Although a large number of characters are described as basic to octopod descriptions (Roper \& Voss 1983), it is the general appearance of the whole animal that the observer first notices. Where several different morphologies exist in animals of the same size and maturity, there are grounds for suspicion that one or more have been induced by variable fixation techniques. The most frequently cited examples are of slender- and squat-bodied forms with otherwise similar anatomies. If two forms consistently have a relaxed or contracted radula support muscle, then similar fixation history is likely. However, if two forms consistently have relaxed and contracted radula support muscles, respectively, then different fixation histories are most likely. If two morphologies both appear to have the radula support muscle in a relaxed state, other factors could be considered responsible for any morphological dichotomy. One possible interpretation is that they represent closely related species. On the basis of anatomy and treatment history (reflected in radula support muscle contraction), the two specimens of Thaumeledone depicted in Fig. 1 and 2 are interpreted as conspecific. 

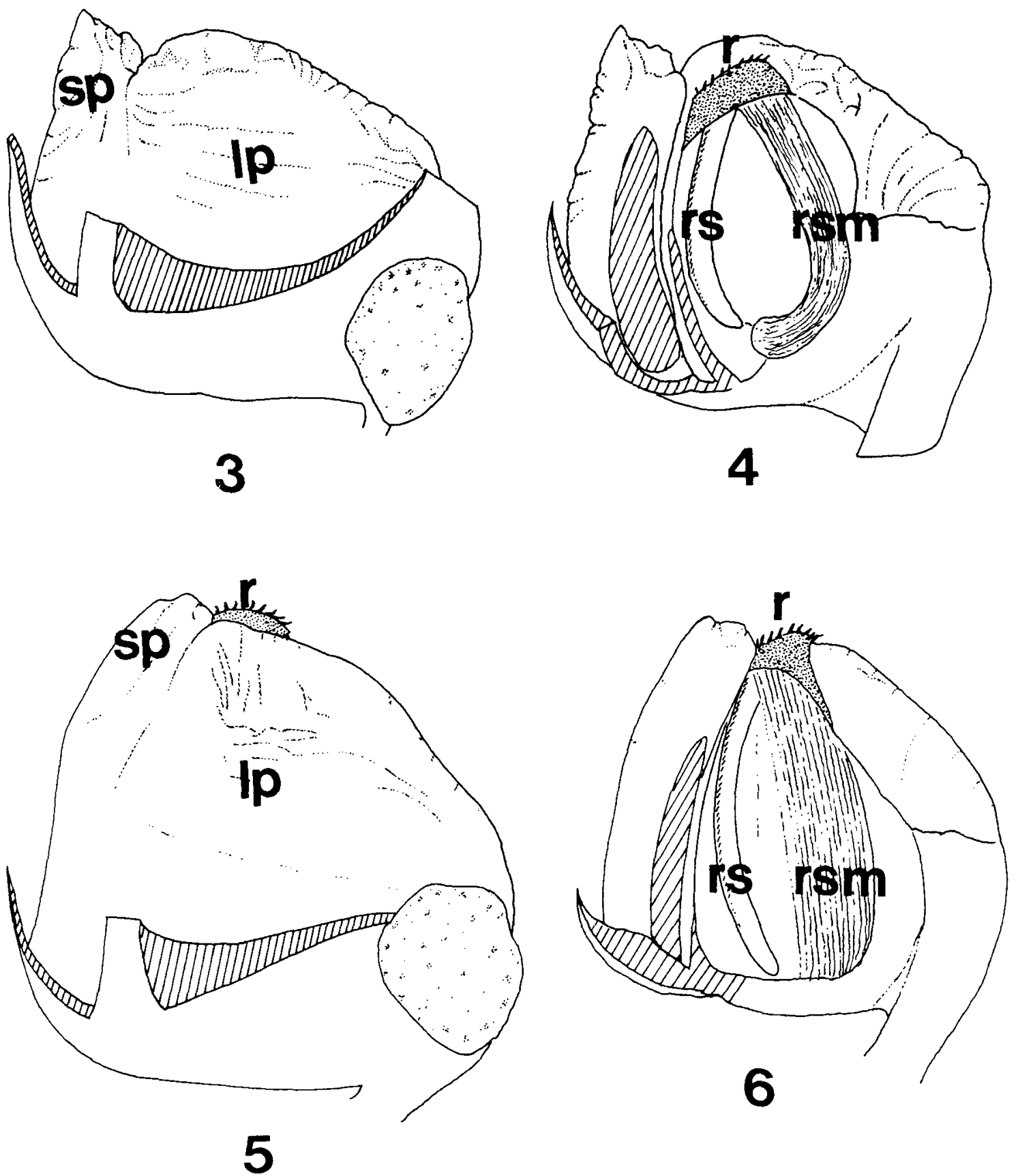

Fig. 3-6 Buccal mass detail of Thaumeledone sp. 3, 4, NZOI Stn S153, specimen fixed live: 3, beaks and lateral muscles removed, lateral palps and salivary papilla exposed, radula sunk between lateral palps; 4 , mid-sagittal view, lateral buccal palp removed, radula sac and contracted radula support muscle exposed. 5, 6, NZOI Stn Z8393, specimen fixed post-mortem (post-thaw), same procedures and views as for (3) and (4): 5, lateral palps, radula, and salivary papilla exposed; 6, radula sac and relaxed radula support muscle exposed. Key: lp, lateral buccal palp; r, radula; rs, radula sac; rsm, radula support muscle; sp, salivary papilla. 
Although the shape and body proportions of a specimen are evidently not characters affected by preservation (Voight 1991), there is a major distinction between methods of preservation and fixation, with manifestly different external morphologies produced in cephalopods subjected to different fixation histories (Andriguetto \& Haimovici 1988). Body morphology viewed in conjunction with the relative contraction of the radula support muscle may prove to be a more valuable tool in differentiating between species than is currently realised.

\section{ACKNOWLEDGMENTS}

I am grateful to Bruce Marshall (Museum of New Zealand), Dennis Gordon (National Institute of Water and Atmospheric Research, New Zealand), Eric Hochburg (Santa Barbara Museum of Natural History, United States), and Clyde Roper (National Museum of Natural History, Smithsonian Institution, United States), for their valuable comments on an earlier draft of the manuscript.

\section{REFERENCES}

Andriguetto, J. M.; Haimovici, M. 1988: Effects of fixation and preservation methods on the morphology of a loliginid squid (Cephalopoda: Mysopsida). American malacological bulletin 6 (2): 213-217.

Nixon, M. 1980: The salivary papilla of Octopus as an accessory radula for drilling shells. Journal of zoology, London 190: 53-57.

Roper, C. F. E.; Voss, G. L. 1983: Guidelines for taxonomic descriptions of cephalopod species. Memoirs of the National Museum of Victoria 44: 48-63.

Voight, J. 1991: Morphological variation in octopod specimens: reassessing the assumption of preservation-induced deformation. Malacologia 33 (1-2): 241-253. 